\title{
Bonnes pratiques des instillations de BCG dans le Traitement des tumeurs vésicales non infiltrant le muscle: Enquête auprès des Urologues Marocains
}

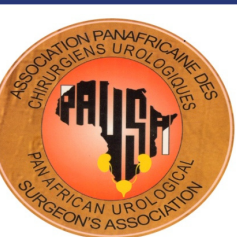

M.A. Touzani $^{1,2}$, I. Ziouziou $^{3}$, S. Regragui $^{1,2}$, T. Karmouni $^{1,2}$, K. Elkhader $^{1,2}$, A. Koutani $^{1,2}$, A. Ibn Attya $^{2}$ Andaloussi, ${ }^{1,2}$

${ }^{1}$ Faculté de Médecine et de Pharmacie, Université Mohammed 5, Rabat, ${ }^{2}$ Service d'urologie «B», Hôpital Ibn Sina, Rabat, ${ }^{3}$ Faculté de Médecine et de Pharmacie, Université Ibn Zohr, Agadir.

\begin{abstract}
Résumé
Introduction: Depuis le premier article publié en 1976 par Morales, les instillations de BCG en intra-vésical ont su s'imposer comme le traitement adjuvant de référence pour la prise en charge des tumeurs vésicales non infiltant le muscle (TVNIM). La réalisation des instillations de $\mathrm{BCG}$ dans de bonnes conditions est essentielle à la réussite de ce traitement.

Objectif: Communiquer les résultats de l'enquête réalisée auprès des urologues marocains avoir une réflexion sur ces résultats et réaliser une revue de la littérature des principales recommandations.

Matériel et méthodes: L'enquête a été réalisée entre le 1er Novembre et le 31 Décembre 2018 auprès d'urologues en formation (internes et résidents) et d'urologues qualifiés (spécialistes et enseignants) via un questionnaire format papier, ou sur Internet $\left(\right.$ Google Forms $\left.{ }^{\circledR}\right)$. Nous nous sommes basés sur les recommandations de huit sociétés savantes internationales.

Résultats: Quatre-vingt-quatorze urologues ont répondu à cette enquête. Près de $20 \%$ ne remettent pas à leur patients de documents explicatifs ou ne réalisent pas une consultation pendant laquelle leur sera expliqué le protocole. Soixante pourcents ne réalisent qu'un seul ECBU au début de la cure, $40 \%$ en réalisent un avant chaque séance. En cas de colonisation, $73 \%$ préfèrent traiter le patient et reporter la séance plutôt que la réaliser sous couverture antibiotique. Dans $68 \%$ des cas, l'urologue réalise lui-même la séance, contre $32 \%$ des cas où il la délègue à un infirmier. Dans près de $90 \%$ des cas, cet infirmier n'a pas bénéficié de formation spécifique. Trente-sept pourcents des praticiens ont déjà été confrontées à gérer des effets indésirables graves.

Conclusion: Il est essentiel de réaliser les instillations selon les bonnes pratiques des sociétés savantes afin de réduire les risques d'évènement indésirable grave qui pourrait amener à arrêter un traitement dont le service médical rendu est important. Mots clés: Tumeur de vessie, Immunothérapie, BCG, Enquête, Bonnes pratiques.

Rec,u le: 17 November 2019, Accepté le: 12 December 2019

Auteur correspondant: Mohammed Alae Touzani, Faculté de Médecine et de Pharmacie, Université Mohammed 5, Rabat, Email: medalaet@gmail.com
\end{abstract}

ISSN: 2090-2379, https://afju.journals.ekb.eg

\section{Introduction}

Depuis le premier article publié en 1976 par Morales ${ }^{[1]}$, les instillations du bacille de Calmette et Guérin (BCG) en intra-vésical ont su s'imposer comme le traitement adjuvant de référence pour la prise en charge des tumeurs de vessie n'infiltrant pas le muscle (TVNIM). Actuellement, les instillations sont indiquées à la fois dans les tumeurs de vessie de risque intermédiaire et de haut risque. La réalisation des instillations de BCG dans de bonnes conditions est essentielle à la réussite de ce traitement. L'objectif de ce travail est de communiquer les résultats de l'enquête que l'on a réalisée auprès des urologues marocains, afin de connaître leurs habitudes concernant la pratique des instillations intra-vésicales de BCG dans le cadre du traitement des TVNIM, mais également avoir une réflexion sur les résultats obtenus, et enfin réaliser une revue de la littérature des principales recommandations de bonnes pratiques des sociétés savantes internationales sur la réalisation des instillations de $\mathrm{BCG}$.

\section{Matériels et méthodes}

L'enquête a été réalisée entre le 1er Novembre et le 31 Décembre 2018 auprès d'urologues en formation (internes et résidents) et d'urologues qualifiés (spécialistes et enseignants) via un questionnaire format papier, ou sur Internet (Google Forms ${ }^{\circledR}$ ). Ce questionnaire était constitué de 36 questions à choix unique. L'enquête était non rémunérée. Les recommandations sur lesquelles 
nous nous sommes basés sont par ordre alphabétique des sociétés savantes : Recommandations du comité de cancérologie de l'Association française d'urologie pour la bonne pratique des instillations endovésicales de $\mathrm{BCG}$ et de mitomycine $\mathrm{C}$ dans le traitement des tumeurs de la vessie n'envahissant pas le muscle (Comité de Cancérologie de l'Association Française d'Urologie, 2012 ${ }^{[2]}$, Recommandations françaises du Comité de Cancérologie de l'AFU : tumeurs de la vessie (Association Française d'Urologie 2013 et 2018-2020 ${ }^{[3,4]}$, Intra-vesical Therapy for Non-Muscle Invasive Bladder Cancer:Nursing Guidelines (Australia \& New Zealand Urological Nurses Society, 2018) ${ }^{[5]}$, Diagnosis and Treatment of Non-Muscle Invasive Bladder Cancer: AUA/SUO Guideline (American Urological Association et Society of Urologic Oncology, 2016) ${ }^{[6]}$, Guidelines for the Administration of Intravesical Therapies (British Association of Urological Nurses, 2010 ${ }^{[7]}$, EAU Guidelines on Non-Muscle-Invasive Bladder Cancer (European Association of Urology, 2018 ${ }^{[8]}$, Evidence-based Guidelines for Best Practice in Urological Health Care : Intravesical instillation (European Association of Urology Nurses, 2015) ${ }^{[9]}$ et NCCN Clinical Practice Guidelines in Oncology:Bladder Cancer (National Comprehensive Cancer Network, 2018) ${ }^{[10]}$.
Pour chaque recommandation émise, le nom de la société ou association sera précisé, ainsi que l'année, suivie du niveau de preuve (LE) et/ou du grade de recommandation (GR) selon le score de SACKETT ${ }^{[1]}$.

\section{Résultats}

\section{Profil des praticiens:}

$\mathrm{Au}$ total, 94 urologues ont répondu à cette enquête : $42 \%$ sont des résidents, $50 \%$ des spécialistes, et $8 \%$ des enseignants. Parmi les urologues confirmés, $50 \%$ ont plus de 10 ans d'exercice. La grande majorité pratique dans le secteur public (73\%) (Tableau 1). Plus de la moitié des urologues sondés $(57 \%)$ indiquent des instillations chez 5 à 20 patients par an. Le questionnaire a pu être rempli sur papier dans quelques cas $(\mathrm{n}=27)$, la majorité l'ayant rempli sur internet de manière anonyme après avoir reçu le lien hypertexte sur messagerie $(n=64)$. La durée moyenne de réponse était de 5,5 minutes. Aucun urologue n'a émis d'objection ou demandé de clarification concernant les questions et les réponses proposées.

Tableau 1: Profil des praticiens sondés

\begin{tabular}{|c|c|c|c|}
\hline \multicolumn{2}{|c|}{ Variables } & \multirow{2}{*}{$\begin{array}{c}\text { Nombre } \\
40\end{array}$} & \multirow{2}{*}{$\frac{\text { Pourcentage }}{42 \%}$} \\
\hline \multirow{3}{*}{ Statut } & Résident & & \\
\hline & Spécialiste & 46 & $50 \%$ \\
\hline & Enseignant & 8 & $8 \%$ \\
\hline \multirow{2}{*}{$\begin{array}{l}\text { Expérience des } \\
\text { urologue confirmés }\end{array}$} & +10 ans & 27 & $50 \%$ \\
\hline & -10 ans & 27 & $50 \%$ \\
\hline \multirow{2}{*}{ Secteur } & Public & 69 & $73 \%$ \\
\hline & Privé & 25 & $27 \%$ \\
\hline
\end{tabular}

\section{Avant la séance:}

Il est à noter que près de $20 \%$ ne remettent pas à leur patients de documents explicatifs ou ne réalisent pas de consultation pendant laquelle leur sera expliqué le protocole, le suivi ainsi que les probables ou possibles effets secondaires. Vingt-trois pourcent ont aménagé un local exclusivement dédié à l'instillation de BCG. Parmi eux, seul un urologue possède une hotte aspirante pour reconstitution du BCG. Parmi ceux qui n'ont pas de local dédié, seuls $25 \%$ attendent la fin de programme journalier (la salle étant dédiée à d'autres soins également), pour débuter les instillations (Figure 1).

Vingt-huit pourcents des urologues réalisent préalablement une estimation du résidu post-mictionnel (RPM), et $8 \%$ demandent au patient d'être à jeun le jour de l'instillation.
En ce qui concerne l'examen cytobactériologique des urines (ECBU), il est demandé par $97 \%$ des praticiens. Parmi eux, $60 \%$ ne réalisent qu'un seul ECBU au début de la cure, contre $40 \%$ qui en réalisent un avant chaque séance. En cas de colonisation, $73 \%$ préfèrent traiter le patient et reporter la séance plutôt que la réaliser sous couverture antibiotique. Aucune antibioprophylaxie n'est administrée aux patients par les $3 \%$ d'urologues qui ne demandent pas d'ECBU. Par contre, $6 \%$ préfèrent donner systématiquement une antibioprophylaxie aux patients, malgré un ECBU stérile (Figure 2).

Quatre-vingt-trois pourcents indiquent souvent une BCG thérapie chez les patients ayant une TVNIM de risque intermédiaire. Pour plus de $80 \%$ des urologues, moins de un patient sur deux n'arrive pas à se procurer le BCG pour des raisons de disponibilité ou financières. 


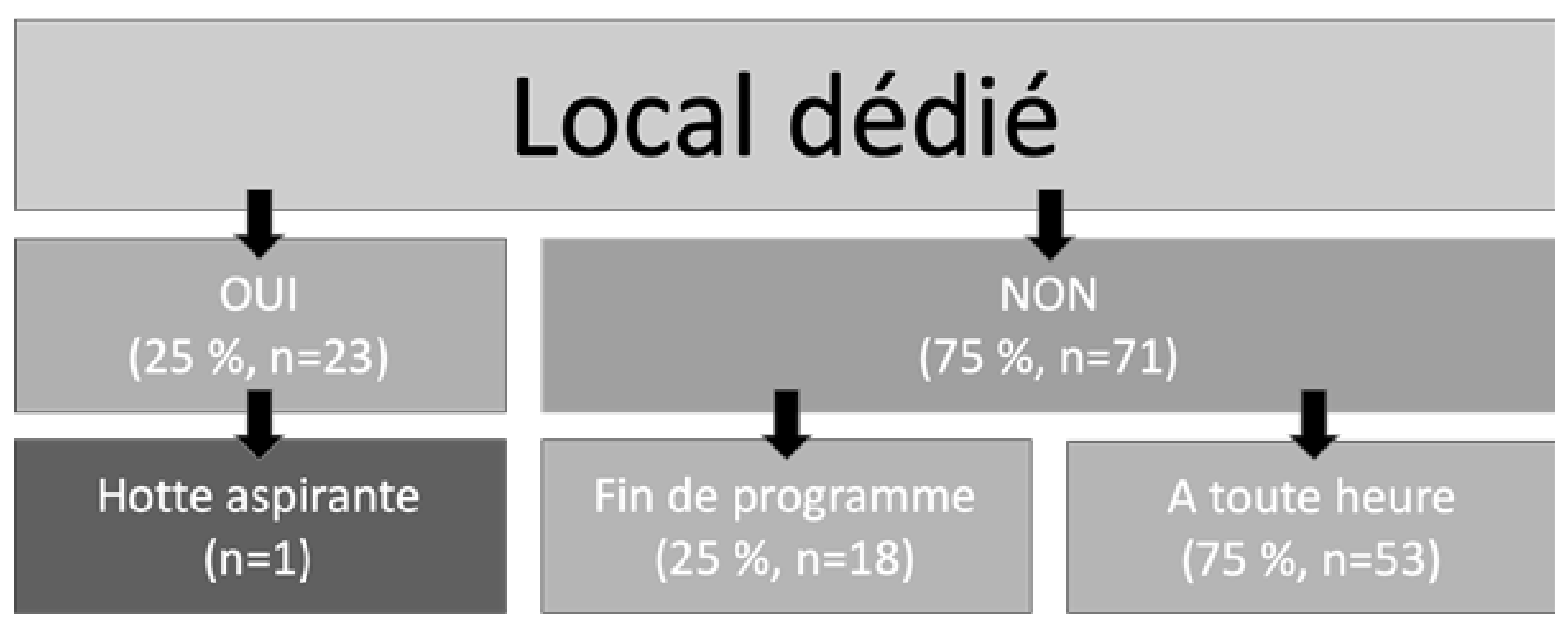

Fig. 1: Local dédié à la BCG thérapie

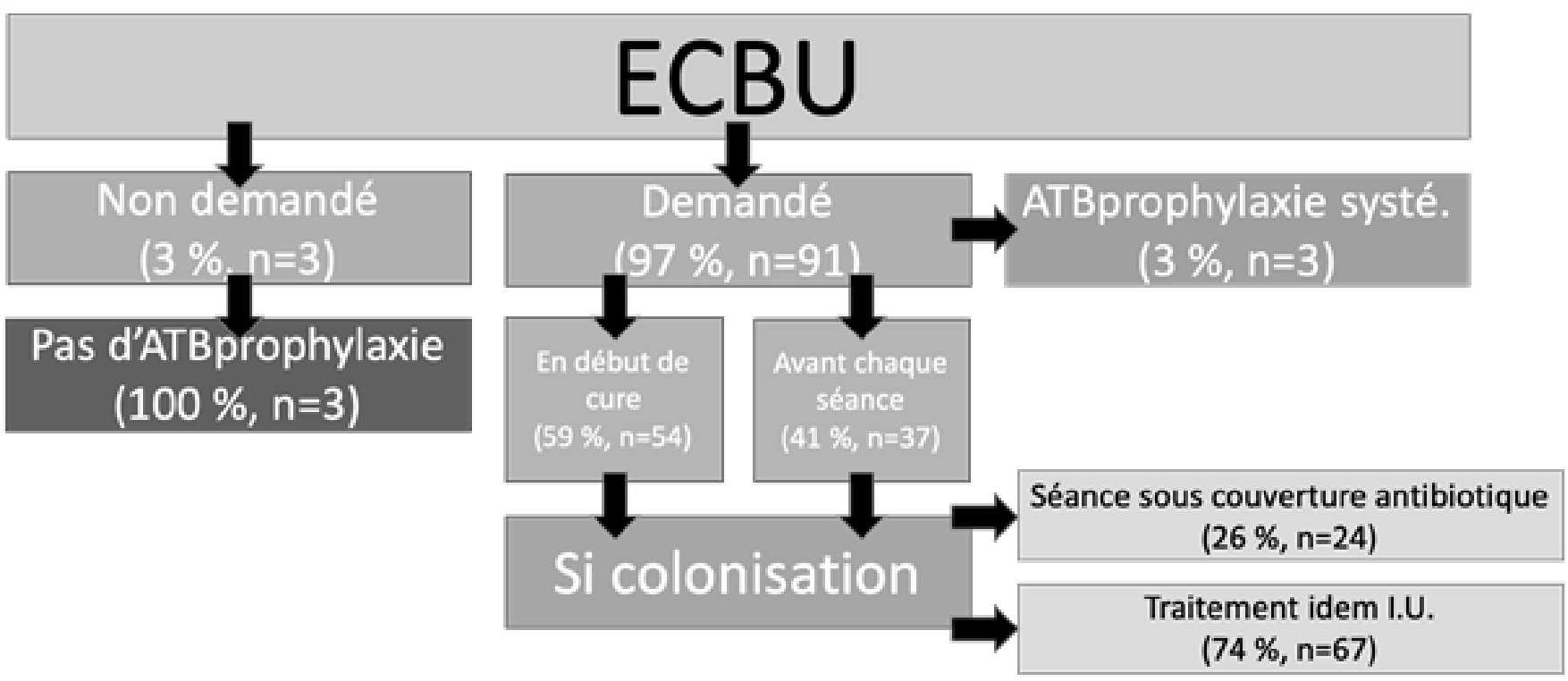

Fig. 2: Gestion de l'ECBU, de l'antibioprophylaxie et du risque infectieux par les praticiens

\section{Pendant la séance:}

Dans $68 \%$ des cas, l'urologue réalise lui-même la séance, contre $32 \%$ des cas où il la délègue à un infirmier. Ceux qui délèguent le plus aux infirmiers sont les résidents $(\mathrm{n}=22 / 30,73 \%)$ et les praticiens du secteur public $(n=27 / 30,90 \%)$. Dans $93 \%$ des cas $(n=28 / 30)$, cet infirmier n'a pas bénéficié de formation spécifique, et parmi eux, seul $53 \%(\mathrm{n}=15 / 28)$ sont assistés par l'urologue lors de l'instillation (Figure 3).

Quatre-vingt pourcents des opérateurs ne sont pas totalement protégés lors de l'acte. Uniquement $22 \%$ recommandent l'utilisation d'une sonde de petit calibre (14 charrière ou moins). Il s'agit dans $76 \%$ de praticiens ayant plus de 5 ans d'expérience ( $n=16 / 21)$. Quatre-vingtseize pourcents reportent la séance en cas de sondage traumatique. Seulement $41 \%$ des praticiens instillent directement à partir de la seringue avec laquelle ils ont réalisé la reconstitution et la dilution du BCG. La majorité (53\%) injecte le produit de façon rapide en moins d'une minute. Quatre-vingt-quatorze désondent le patient systématiquement en fin d'injection.

En ce qui concerne la durée de contact, $65 \%$ demandent aux patients de garder le produit $2 \mathrm{~h}$ dans leur vessie. Aucun praticien ne demande au patient de le garder plus que 2 h. Près de $7 \%(n=6)$ ne demandent au patient de garder le produit qu'une heure ou moins. Pour la position, 84 $\%$ demandent aux patients de réaliser un changement de position à intervalles réguliers après instillation, contre $11 \%$ qui autorisent un lever au bout de quelques minutes.

Soixante et un pourcents ont dans leur local un réceptacle destinée à recevoir les déchets d'activités de soins à risques infectieux (DASRI). 


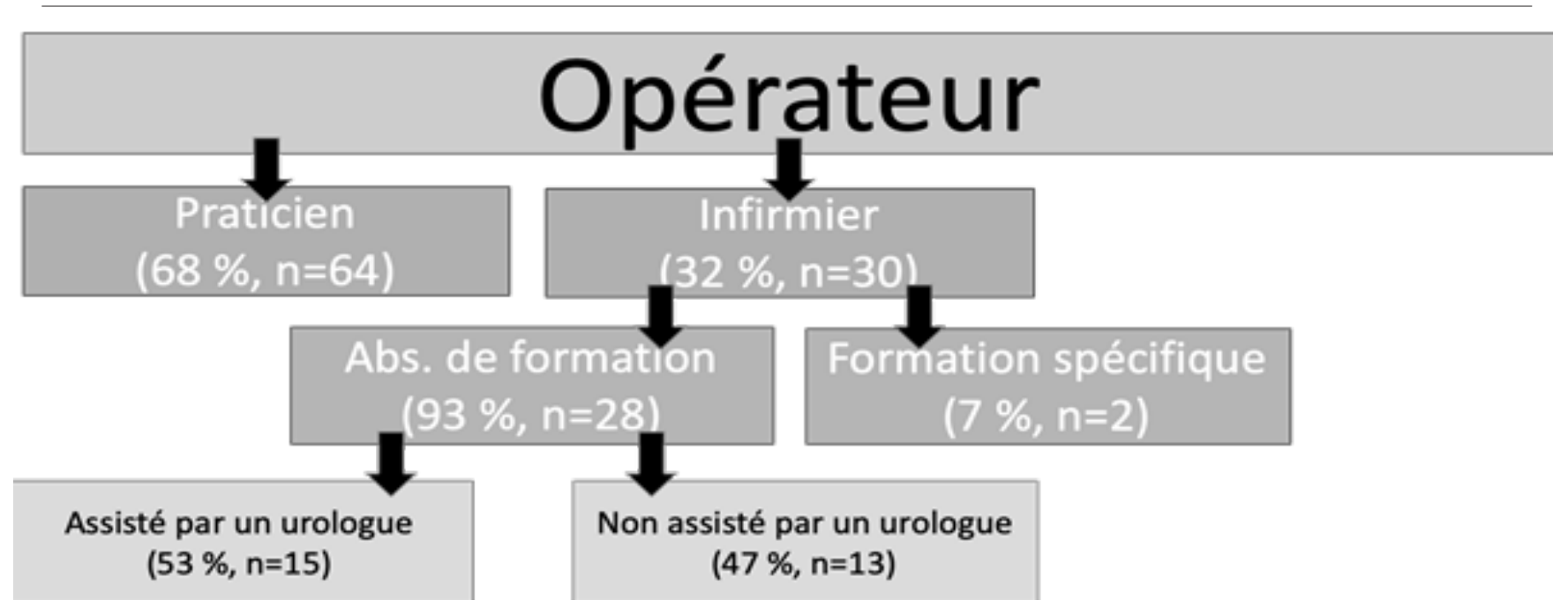

Fig. 3: Données sur les opérateurs réalisant la BCG thérapie

\section{Après la séance:}

Vingt-neuf pourcents préconisent d'uriner en position assise, $62 \%$ demandent au patient de verser de l'eau de Javel dans la cuvette après avoir uriné, dont $71 \%$ $(n=42 / 59)$ seulement le jour même. Soixante-six et $84 \%$ recommandent respectivement aux patients de prendre systématiquement leur température après la séance et de recenser les évènements indésirables survenant après la séance. Seuls $8 \%$ demandent à leurs patients d'éviter les rapports sexuels non protégés pendant les 48 heures suivant l'instillation. Enfin, $37 \%$ des praticiens ont déjà été confrontées à gérer des effets indésirables graves (ayant nécessité une hospitalisation et l'introduction d'un traitement antituberculeux). Dans $78 \%(n=7 / 24)$, il s'agissait de praticiens ayant plus de 5 ans d'expérience. Des cas de tuberculose ganglionnaire, miliaire tuberculeuse, prostatite, orchite, hépatite ictérique, de masses pelviennes, avec un cas de décès ont été cités.

\section{Discussion}

\section{Représentativité du panel sondé:}

Quatre-vingt-quatorze urologues ont répondu à cette enquête. Selon les estimations, ce chiffre représente près de $20 \%$ à $30 \%$ des urologues du Maroc. La répartition entre statut et années d'expérience est également homogène et représentative.

\section{Le local:}

Comme le montrent les résultats, il est difficile de concevoir, que ce soit pour un cabinet privé ou une structure publique, qu'une salle soit dédiée exclusivement aux instillations de BCG. Ainsi, cette salle devrait être au moins être bien ventilée et ensoleillée, permettant ainsi un contrôle environnemental d'éventuelles contaminations ${ }^{[12]}$. Il serait préférable de réaliser l'instillation en fin de programme journalier afin de minimiser ce risque. Les recommandations précisent que la reconstitution du BCG et son instillation ne doivent pas être réalisés dans les zones ou sont administrés des traitements par voie intraveineuse, ceci étant associé à plus d'infections nosocomiales chez les patients recevant ces derniers (EAUN 2015/LE:3/GR:B) ${ }^{[9]}$. La présence d'une hotte aspirante pour reconstitution serait également un bon moyen de protection, cependant son coût constitue une limite. Ce local devrait disposer d'un réceptacle pour recueil des produits tranchants et un autre pour les DASRI. Tout le consommable ayant été utilisé pour l'instillation devra y

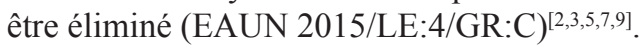

\section{La préparation du patient:}

Lorsque l'indicationd'un traitement par instillation de BCG est posée, le patient doit être informé du traitement qu'il va recevoir, avec une explication de la technique, du rythme prévu et de la durée, du coût, des résultats attendus, du risque d'échec, des modalités de surveillance, et des complications éventuelles. Une documentation devrait également être remise au patient pour lui permettre d'en discuter avec sa famille (EAUN 2015/LE:4/GR:C) $)^{[2,4,5,7,9]}$.

Concernant la prescription, il n'y a pas de données suffisantes sur la supériorité d'une souche par rapport à l'autre (EAU 2018/LE:2a) ${ }^{[2,6,8]}$. Chaque séance correspond à une instillation de 108 à 109 unités formant colonies de bactéries lyophilisées. Il est possible de réduire la dose à $1 / 3$ de la dose normale chez certains patients présentant des effets indésirables locaux mineures et persistants, mais au risque d'augmenter le taux de récidives (EAU 2018/LE:1b) ${ }^{[7]}$. En dessous de 1/3, il y a perte de l'efficacité sans diminution des effets secondaires (CCAFU 2018-2020/LE:1) (EAU 2018/LE:1b) ${ }^{[4,8]}$. 
Un délai minimum de 2-3 semaines, jusqu'à 6 semaines, doit séparer la RTUV (ou éventuelle biopsie prostatique) de la première instillation afin de minimiser le passage systémique (CCAFU 2018-2020/LE:1) (EAUN 2015/LE:3) (NCCN 2018/LE:2a) $)^{[2,4,5,7,9,10] .}$

Il serait intéressant d'évaluer le RPM avant de débuter les instillations. En effet, les patients ayant un RPM significatif, étant plus en contact avec le BCG, serait plus enclin à présenter des signes générau ${ }^{[5]}$.

Seule l'AFU recommande la réalisation d'un bilan pré-thérapeutique constitué de:numération formule sanguine, ionogramme, bilan hépatique, et radiographie de thorax, avant le début des instillations (CCAFU BCG 2012/LE:4) ${ }^{[2]}$. La réalisation d'un ECBU est optionnelle (CCAFU 2018-2020/GR:B) $^{[4]}$ et la découverte d'une leucocyturie et/ou la bactériurie asymptomatique ne sont pas des contre-indications et ne nécessitent pas de traitement (CCAFU 2018-2020/GR:B) (EAU 2018/LE:3) $)^{[4,8]}$. Par contre, les anglophones préfèrent réaliser systématiquement un ECBU soit en début de cure $^{[5]}$, soit avant chaque séance ${ }^{[7]}$, avec report de la séance, prescription d'un traitement antibiotique curatif adapté à l'antibiogrammes, et réalisation d'une bandelette le jour de l'instillation en cas de colonisation ( $\mathrm{NCCN}$ 2018/LE:2a ${ }^{[5,7,10]}$. Une antibioprophylaxie à base quinolone était discutée par le CCAFU, mais n'est plus de mise depuis 2016 (CCAFU 2013-2016/LE:2) ${ }^{[3]}$. Elle aurait pour but de diminuer les effets indésirables graves (CCAFU 2013-2016/LE:2) (CCAFU BCG 2012/LE:2) $)^{[2,3]}$. Cependant, au vu de l'activité antituberculeuse des quinolones, leur administration pourrait diminuer la stimulation de la réaction immunitaire, et donc diminuer les chances de réussite du traitement ${ }^{[2,9]}$. Ainsi, la prescription d'antibiotiques durant toute la durée des instillations doit être réalisée avec précaution ${ }^{[9]}$.

\section{Avant l'instillation:}

Le patient devrait être interrogé, via une check-list, sur son identité, ainsi que la tolérance de la dernière instillation à la recherche d'éléments contre-indiquant la poursuite du traitement ou son report ${ }^{[2]}$ (Tableau 2).

Le produit doit être conservé dans un réfrigérateur dans une température comprise entre 2 et $8^{\circ} \mathrm{C}$, et à l'abri de la lumière ${ }^{[7]}$. En cas de reconstitution, il doit être instillé dans les 2 heures $^{[5,7]}$.

Le patient doit limiter, voire suspendre, les apports en liquide les 4 à 8 heures précédant l'instillation, afin de réduire la production d'urine, permettant ainsi de réduire la dilution du produit mais également d'augmenter les chances du patient de retenir le produit en intra-vésical pendant la durée nécessaire (CCAFU BCG 2012/LE:3) (EAUN 2015/LE:4) ${ }^{[2,5,7,9]}$. Pour les mêmes raisons, la prise de diurétiques doit être espacée de quelques heures par rapport à l'instillation ${ }^{[5,7]}$.

Tableau 2: Contre-indications aux instillations de BCG selon les différentes sociétés

\begin{tabular}{|c|c|c|}
\hline \multirow{4}{*}{ AFU [4] } & Antécédent de réaction systémique au $\mathrm{BCG}$ & $\begin{array}{l}\text { Persistance d'effets secondaires liés } \\
\text { à la précédente instillation }\end{array}$ \\
\hline & Déficit immunitaire sévère & Infection des voies urinaires \\
\hline & Cystite radique & $\begin{array}{l}\text { Absence ou incertitude de l'intégrité } \\
\text { de l'urothélium ( } 2 \text { premières semaines }\end{array}$ \\
\hline & Tuberculose active & $\begin{array}{c}\text { suivant la RTUV, Hématurie } \\
\text { macroscopique, sondage traumatique) }\end{array}$ \\
\hline \multirow{7}{*}{$\begin{array}{l}\text { ANZUNS } \\
\quad[5]\end{array}$} & Tuberculose active & \\
\hline & Antécédent de tuberculose & \\
\hline & HIV positif & \\
\hline & Actuelle radiothérapie ou chimiothérapie & \\
\hline & Troubles cognitifs sans assistance & \\
\hline & Femme enceinte et allaitement & \\
\hline & Enfants & \\
\hline EAU [8] & $\begin{array}{c}\text { Absence ou incertitude de l'intégrité de l'urothélium } \\
\text { (2 premières semaines suivant la RTUV, Hématurie } \\
\text { macroscopique, sondage traumatique) }\end{array}$ & \multirow[t]{2}{*}{ Déficit immunitaire } \\
\hline$(\mathrm{GR}: \mathrm{C})$ & Infection des voies urinaires & \\
\hline
\end{tabular}




\begin{tabular}{|c|c|}
\hline \multirow{5}{*}{ EAUN [9] } & Antécédent de réaction systémique au $\mathrm{BCG}$ \\
\hline & Actuelle radiothérapie \\
\hline & Tuberculose active \\
\hline & Infection des voies urinaires \\
\hline & Hématurie macroscopique \\
\hline & Femme enceinte et allaitement \\
\hline NCCN [10] & $\begin{array}{c}\text { Absence ou incertitude de l'intégrité de l'urothélium } \\
\text { (2 premières semaines suivant la RTUV, Hématurie } \\
\text { macroscopique, sondage traumatique) }\end{array}$ \\
\hline
\end{tabular}

(LE : 2a) Bactériurie

\section{L'instillation:}

L'instillation doit être réalisée par le médecin, ou par le personnel infirmier, à condition que le personnel soit formé par un praticien expérimenté. L'infirmier doit savoir sonder de façon aseptique et atraumatique, connaitre le produit à instiller, le rythme des instillations, les contreindications, les effets indésirables, et la gestion des déchets contaminés (CCAFU BCG 2012/LE:4) (EAUN 2015/LE:4/GR:C) $)^{[2,7,9]}$.

La reconstitution nécessite le port de moyens de protection : surblouse de protection à manches longues, gants stériles, lunettes de protection et masque de protection classe FFP2 ou N95 $5^{[2,5,7,9]}$. La reconstitution est contre-indiquée chez le personnel immunodéprimé ou les femmes enceintes ${ }^{[2]}$. La reconstitution doit se faire par du sérum physiologique isotonique ${ }^{[7]}$.

Il est indiqué d'utiliser une sondé urétrale de petit calibre (EAUN 2015/LE:4/GR:C), avec revêtement hydrophile (EAUN 2015/LE:3/GR:B), lubrifiée (EAUN 2015/LE:2a/GR:B), avec raccord de type «luer-lock » à 3 voies (EAUN 2015/LE:4/GR:C), afin de réduire le risque de sondage traumatique, d'empêcher une injection trop rapide de produit en intra-vésical et minimiser le risque d'exposition au produit ${ }^{[5,7,9]}$. Un sondage traumatique constitue une contre-indication formelle à la poursuite du traitement (EAUN 2015/LE:3) $)^{[2,5,7,9]}$. La séance devra être reportée d'au moins une semaine (EAUN 2015/ LE:3) (NCCN 2018/LE:2a) ${ }^{[2,9,10]}$. En cas de traumatisme avéré après instillation, il faudra procéder à un rinçage de la vessie au sérum physiologique suivie d'une prise d'ofloxacine (400 mg par jour) pendant 8 jours (CCAFU BCG 2012/LE:3 $)^{[2]}$.

L'injection du produit doit se faire de manière lente et sans forcer, de préférence par gravité, afin d'éviter de stimuler des contractions vésicales ${ }^{[2,5,7]}$. Il est nécessaire d'attendre que la vessie se vide avant d'injecter le produit. La sonde doit être retirée après injection, sauf s'il s'agit d'un patient ayant un RPM significatif ou d'un patient incontinent ${ }^{[2,5,7]}$. Dans ces 2 cas, la sonde sera clampée la durée de l'instillation, puis retirée après vidange vésicale $^{[5,7]}$.

Concernant la durée d'exposition à la préparation, alors que l'AFU préconise de garder la suspension au minimum $2 \mathrm{~h}^{[2]}$, les autres sociétés recommandent une durée minimale de $1 \mathrm{~h}$, et de préférence $2 \mathrm{~h}$, sans les dépasser ${ }^{[5,7,9]}$. Il n'y a aucune preuve concernant la supériorité de l'efficacité du traitement en cas de changement de position à intervalles réguliers rapport au décubitus dorsal strict ou à la position debout ${ }^{[2,5,7,9]}$. L'anatomie de la vessie semi-pleine, fait que, en l'absence d'air, toute la paroi sera en contact avec le produit quelle que soit la position adoptée.

\section{Après l'instillation:}

Il sera recommandé aux patients d'uriner en position assise, directement dans la cuvette, dans les 6 heures qui suivent l'instillation afin d'éviter toute contamination ${ }^{[2,5,7,9]}$. La neutralisation des urines par de l'eau de Javel est sujette à controverse. Alors que les anglophones recommandent, sans preuve, de verser l'équivalent de 1 à 2 verres d'eau de Javel non diluée immédiatement après la miction, attendre 15 avant de tirer la chasse d'eau ${ }^{[5,7,9]}$, l'AFU ne le recommande pas $^{[2]}$. Une hyperhydratation devra être débutée par le patient des lors qu'il urine (CCAFU BCG 2012/LE:3 $)^{[2,5,7,9]}$. Les recommandations ne précisent pas le volume journalier; sa durée sera au minimum de 48 heures ${ }^{[2]}$, jusqu'à 7 jours ${ }^{[5]}$. Les rapports sexuels devront être protégés pendant au moins 2 à 7 jours suivant l'instillation, et il est recommandé aux femmes d'éviter toute grossesse suivant les 6 mois $^{[5,7,9]}$.

\section{Conclusion}

Cette enquête a permis de faire le point sur la pratique des instillations de BCG au Maroc. Il est essentiel de réaliser les instillations selon les bonnes pratiques des sociétés savantes afin de réduire le risque d'évènement indésirable grave qui pourrait amener à arrêter un traitement dont le service médical rendu est important. De nombreux 
points ne font pas encore l'objet de recommandations, et l'on espère que ce travail puisse aider à y remédier. La réalisation d'une fiche informative en arabe semble être le meilleur moyen d'informer le patient des principes et risques du traitement.

\section{Approbation éthique et consentement à participer}

Ce travail a été réalisé après avoir eu l'aval des encadrants et de l'Association Marocaine d'Urologie.

\section{Intérêts concurrents}

Les auteurs déclarent n'avoir aucun conflit d'intérêts en relation avec cet article.

\section{Source de financement}

Aucune.

\section{Contributions des auteurs}

MAT: Élaboration du questionnaire, étude statistique et revue de la littérature.

IZ: Élaboration du questionnaire et étude statistique.

SR: Distribution du questionnaire.

TQ, KE, AK et AIAA: Correction et finalisation du travail.

\section{Références}

[1] Morales A, Eidinger D, Bruce AW. Intracavitary bacillus Calmette-Guerin in the treatment of superficial bladder tumors. J Urol 1976; 116: 180-83

[2] Rouprêt M, Neuzillet Y, Larré S, Pignot G, Coloby $\mathrm{P}$, Rebillard X, et al. Recommandations du Comité de cancérologie de l'Association française d'urologie (CC$\mathrm{AFU}$ ) pour la bonne pratique des instillations endovésicales de $\mathrm{BCG}$ et de mitomycine $\mathrm{C}$ dans le traitement des tumeurs de la vessie n'envahissant pas le muscle (TVNIM). Prog Urol 2012;22:920-31.

[3] Pfister C, Roupret M, Neuzillet Y, Larre S, Pignot G, Quintens $\mathrm{H}$, et al. Recommandations en onco-urologie 2013 du CCAFU : Tumeurs de la vessie. Prog Urol 2013;23:S105-25.

[4] Rouprêt M, Neuzillet Y, Pignot G, Compérat E, Audenet F, Houédé N, et al. French ccAFU guidelines - Update 2018-2020: Bladder cancer. Prog Urol. 2018 Nov;28(12S):S46-S78
[5] Intra-vesical therapy for Non-Muscle Invasive Bladder Cancer (NMIBC) Nursing Guidelines [Internet]. Australian and New Zealand Urology Nurses Society (ANZUNS). 2018 [Cité le 18 Février 2019] Disponible sur : http://www. anzuns.org/wp-content/uploads/2018/03/Intra-vesicalTherapy-for-NMIBC-Guidelines.pdf

[6] Chang SS, Boorjian SA, Chou R et al. Diagnosis and Treatment of NonMuscle Invasive Bladder Cancer: AUA/ SUO Guideline. J Urol 2016; 196: 1021-9

[7] Guidelines for the Administration of Intravesical Therapies. British Association of Urological Nurses (BAUN). 2010 [Cité le 18 Février 2019] Disponible sur : http://www.baun.co.uk/files/3713/2438/5251/ BSO Guidelines_Feb_2011.pdf

[8] Babjuk M, Burger M, [Compérat E, Gontero P, Mostafid A H, Palou J et al. EAU Guidelines on Nonmuscle-invasive Bladder Cancer: Update 2018 [Internet]. European Association of Urology (EAU). 2018 [Cité le 18 Février 2019] Disponible sur : http://uroweb.org/guideline/ non-muscle-invasive-bladder-cancer/

[9] Vahr S, De Blok W, Love-Retinger N, Thoft Jensen $\mathrm{B}$, Turner B, Villa $\mathrm{G}$ et al. Intravesical instillation with mitomycin $\mathrm{C}$ or bacillus Calmette-Guérin in non-muscle invasive bladder cancer Evidence-based Guidelines for Best Practice in Urological Health Care [Internet]. European Association of Urology Nurses (EAUN) 2015 [Cité le 18 Février 2019] Disponible sur : http://nurses. uroweb.org/guideline/intravesical-instillation-withmitomycin-c-or-bacillus-calmette-guerin-in-non-muscleinvasive-bladder-cancer/

[10] Flaig TW, Spiess PE, Agarwal N, et al. NCCN guidelines insights: bladder cancer, version 5.2018. J Natl Compr Canc Netw 2018; 16: 1041-53

[11] Phillips B, Ball C, Sackett D, et al. Oxford Centre for Evidence-based Medicine - levels of evidence. Oxford Centre for Evidence-based Medicine Web site. http://www. cebm.net/oxford-centreevidence-based-medicine-levelsevidence-march-2009/. Updated March 2009

[12] Lee JY. Tuberculosis Infection Control in HealthCare Facilities: Environmental Control and Personal Protection. Tuberc Respir Dis (Seoul) 2016; 79: 234 40. 


\title{
Good practices of instillations of BCG for the Treatment of TVNIM: Survey of Moroccan Urologists
}

\begin{abstract}
Introduction: Since the first article published in 1976 by Morales, intravesical BCG instillations became the standard adjuvant treatment for the management of TVNIM. Performing BCG instillations in good conditions is essential to the success of this treatment.

Objective: To communicate the results of the survey carried out among Moroccan urologists in order to know their habits concerning the practice of intravesical instillations of BCG, to have a reflection on these results and make a summary of society guidelines on BCG instillations.

Material and Methods: The survey was conducted between November 1 and December 31, 2018 with urologists in training (interns and residents) and qualified urologists (specialists and teachers) via a paper questionnaire, or on the Internet (Google Forms $^{\circledR}$ ). We based our work on the guidelines of eight international urological associations.

Results: Ninety-four urologists responded to this survey. Nearly $20 \%$ do not give explanatory documents to their patients or do not perform a consultation during which the protocol will be explained to them. Sixty percent do only one ECBU at the beginning of the treatment, $40 \%$ make one before each session. In the case of colonization, $73 \%$ prefer to treat the patient and postpone the session rather than carry it under antibiotic coverage. In $68 \%$ of the cases, the urologist carries out the session himself, as against $32 \%$ of the cases where he delegates it to a nurse. In almost $90 \%$ of cases, this nurse did not receive specific training. Thirty-seven percent of practitioners have already experienced serious side effects.

Conclusion: It is essential to perform instillations according best practice guidelines in order to reduce the risk of a serious adverse event that could lead to stop a treatment whose medical service is important.
\end{abstract}

Keywords: Bladder cancer, Immunotherapy, BCG, Survey, Best practices. 